\title{
Realinhamento do eixo mecânico da extremidade distal do fêmur com fixação externa híbrida modificada*
}

\author{
Realignment of the mechanical axis of the distal femur \\ extremity with modified hybrid external fixation
}

\author{
Paulo Henrique Bortolin ${ }^{1}$, José B. Volpon ${ }^{2}$
}

\section{RESUMO}

Objetivo: Avaliar os resultados da retificação do eixo mecânico dos membros inferiores por meio da correção aguda de deformidade distal do fêmur usando um tipo de fixação externa híbrida dos fragmentos. Métodos: Quinze joelhos de 11 pacientes com deformidade na extremidade distal do fêmur foram submetidos à correção do ângulo frontal do joelho por apresentarem deformidades em varo ou valgo, sem desvios no plano sagital. A técnica incluiu planejamento pré-operatório, osteotomia percutânea na metáfise distal do fêmur, correção aguda da deformidade angular associada à translação, e fixação da osteotomia por fixador externo híbrido com montagem modificada. A avaliação radiográfica foi realizada em períodos intermediários ao

* Trabalho realizado no Setor de Ortopedia Pediátrica do Hospital das Clínicas da Faculdade de Medicina de Ribeirão Preto da Universidade de São Paulo - USP - Ribeirão Preto (SP), Brasil.

1. Mestre, Médico Assistente do Setor de Ortopedia Pediátrica do Hospital das Clínicas da Faculdade de Medicina de Ribeirão Preto da Universidade de São Paulo - USP - Ribeirão Preto (SP), Brasil.

2. Professor Titular e Responsável pelo Setor de Ortopedia Pediátrica do Hospital das Clínicas da Faculdade de Medicina de Ribeirão Preto da Universidade de São Paulo - USP - Ribeirão Preto (SP), Brasil.

Endereço para correspondência: José B. Volpon, Rua São José, 655, apto. 901, Centro - 14010-160 - Ribeirão Preto (SP), Brasil.

E-mail: jbvolpon@fmrp.usp.br

Recebido: 10.4.08. Revisão: 20.6.08. Reapresentação: 20.10.08. Aprovado: 30.10.08.

Copyright RBO2008 longo dos seis meses do período pós-operatório, com acompanhamento clínico médio de 17 meses. $R e$ sultados: Todas as osteotomias consolidaram-se, em tempo médio de três meses. Em apenas um caso houve complicação clínica significativa representada por fratura após a retirada do fixador, o que levou a nova colocação. $O$ ângulo mecânico femoral distal lateral (mLDFA) retornou aos valores normais em nove de 15 joelhos, com média de 71,5\%. Em quatro extremidades, desvios residuais leves do eixo mecânico foram causados por deformidade tibial e em uma por deformidade em varo da extremidade proximal do fêmur. Conclusão: A técnica de correção do desvio do segmento distal do fêmur, por osteotomia distal compensada pela translação do fragmento, foi adequada para restaurar o eixo mecânico do membro inferior e o fixador externo híbrido com a montagem modificada foi eficiente para manter as correções agudas obtidas no momento da cirurgia e propiciar a consolidação óssea.

Descritores - Fixadores externos; Fêmur/anatomia \& histologia; Osteotomia/métodos; Técnica de Ilizarov

\section{ABSTRACT}

Objective: To evaluate the results achieved with the correction of the mechanical axis of lower limbs through acute correction of a distal femur deformity using a hybrid type of external fixation to fixate the fragments. Methods: 15 knees of 11 patients with 
deformity in the distal femur extremity were submitted to correction of the frontal angle of the knee because they had either varus or valgus deformity, without displacement in the sagittal plan. The technique involved pre-operative planning, percutaneous osteotomy in the distal femoral metaphysis, acute correction of the angular deformity associated to translation, fixation of the osteotomy using a hybrid external fixator with a modified assembly. Radiographic evaluation was performed in intermediary periods along the six-month period after surgery, with mean clinical follow-up of 17 months. Results: Union was achieved in all of the osteotomies in a mean time of three months. Only one case presented a significant clinical complication represented by a fracture after the fixator was removed, and a new fixator had to be placed. The lateral distal femoral mechanical angle (mLDFA) returned to normal values in 9 of the 15 knees, with a mean of $71.5 \%$. In four extremities, slight residual displacement of the mechanical axis was caused by tibial deformity and one by a varus deformity in the proximal femur extremity. Conclusion: The technique of correcting the displacement in the distal segment of the femur using distal osteotomy offset by the fragment translation was adequate to recover the mechanical axis of the lower limb, and the hybrid external fixator with the modified assembly was efficient to maintain the acute corrections achieved by the surgery and to provide for bone union.

\section{Keywords - External fixators; Femur/anatomy \& histology; Osteotomy/methods; Ilizarov technique}

\section{INTRODUÇÃO}

As deformidades dos membros inferiores no plano frontal destacam-se por sua freqüência na população pediátrica em atendimento em hospital terciário e podem ser a expressão clínica de inúmeras afecções ortopédicas, metabólicas ou traumáticas. Sua importância aumenta à medida que, na idade adulta, o desenvolvimento e a progressão da osteoartrose do joelho podem correlacionar-se com o grau da deformida$\mathrm{de}^{(1-3)}$.
No planejamento de uma osteotomia corretiva de qualquer tipo é importante determinar a localização e magnitude do desvio, para que, idealmente, a correção seja feita no ápice da deformação, pois, dessa forma, o efeito corretivo é maior, mais fisiológico e evita-se criar deformidade secundária na tentativa de correção da preexistente. Entretanto, essa situação ideal nem sempre é alcançável, seja pela presença da cartilagem de crescimento, seja pelo tamanho do fragmento ou qualidade do osso. Assim, se a osteotomia não puder ser realizada no mesmo local da deformidade, será necessário acrescentar translação entre os fragmentos para, além de garantir a restauração do eixo mecânico, evitar a má orientação da articulação do joelho $^{(4-5)}$.

Outro problema existente nesses casos é a dificuldade adicional em fixar o pequeno fragmento distal, especialmente no esqueleto imaturo e em osso previamente afetado, principalmente por condições osteometabólicas. Os dispositivos de fixação interna como as placas, retas ou anguladas, são geralmente inadequados, mesmo em adultos, por dificultar a translação dos fragmentos ${ }^{(6-8)}$. Os fixadores externos unilaterais não apresentam estabilidade suficiente no plano sagi$\operatorname{tal}^{(5)}$ e os fixadores externos circulares tipo Ilizarov são bastante desconfortáveis quando utilizados na coxa. Autores relataram o uso temporário do fixador externo para realizar a correção, com a fixação definitiva por haste intramedular, método que não pode ser utilizado no esqueleto imaturo ${ }^{(9-10)}$.

O fixador externo híbrido é utilizado na estabilização de fraturas metafisárias e/ou articulares em adultos e, potencialmente, apresenta propriedades mecânicas adequadas para a fixação de osteotomias da extremidade distal do fêmur, visto que sua parte circular provê fixação estável e multiplanar do pequeno fragmento distal; angulação e translação podem ser facilmente acrescentadas durante a cirurgia, propiciando controle radiográfico preciso, sendo que a parte monoplanar agrega maior conforto quando comparada com as montagens convencionais de fixadores circulares aplicados na coxa. Faria et al descreveram modificação simplificada da montagem do fixador externo híbrido obtida pela associação de elementos usados nos 
fixadores AO e Ilizarov. Eles usaram o método para fixação de fraturas proximais e distais de tíbia e, a partir dessa idéia, empregamos a mesma montagem para fixação de osteotomias distais do fêmur ${ }^{(11)}$.

Assim, o objetivo desta investigação foi avaliar os resultados da correção do eixo mecânico dos membros inferiores por meio da correção aguda de deformidade distal do fêmur e usando um tipo de fixação externa híbrida dos fragmentos.

\section{MÉTODOS}

Foram submetidos à correção de eixo mecânico dos membros inferiores 11 pacientes (15 fêmures) com desvio angular frontal nos joelhos, de diferentes etiologias, entre 2000 e 2003, no Hospital das Clínicas da Faculdade de Medicina de Ribeirão Preto - USP. O projeto foi aprovado pela Comissão de Ética Institucional. O modelo de estudo foi prospectivo, com os seguintes critérios de inclusão: deformidade localizada na extremidade distal do fêmur, sem desvio no plano sagital, desvio do eixo mecânico maior do que $15 \mathrm{~mm}$, com associação ou não a outras deformidades (tíbia, região proximal do fêmur); população constituída de indivíduos esqueleticamente imaturos ou adolescentes; sem necessidade de equalização de comprimento e seguimento mínimo por tempo predeterminado de seis meses.

Entre os pacientes, sete eram do sexo feminino e quatro do masculino, sendo cinco portadores de seqüela de raquitismo hipofosfatêmico, dois com seqüela de displasia espondilometafisária, dois com seqüela de artrite séptica do joelho, um com sequiela de lúpus eritematoso sistêmico e um com joelho valgo idiopático.

A média de idade na época da cirurgia foi de 11 anos e cinco meses (3-20 anos), mediana de 14 anos, sendo que no fêmur a cartilagem de crescimento distal estava fechada em dois casos. Todos os pacientes eram deambuladores comunitários, mas sete referiam dificuldade para caminhar devido à deformidade.

Dos fêmures, oito apresentavam desvio em varo e sete em valgo e, em todos eles, exclusivamente extraarticular. A deformidade na região distal do fêmur estava associada à deformidade tibial em sete pacientes e, em quatro extremidades, foi realizado o alinhamento da tíbia com osteotomia e fixação com placa de compressão, no mesmo tempo cirúrgico da correção femoral. Deformidades da região proximal do fêmur (mau alinhamento da articulação do quadril) estavam presentes em oito fêmures, porém nenhum deles foi submetido ao alinhamento da região proximal do fêmur por serem alterações discretas, sem repercussão clínica na marcha.

A avaliação clínica foi realizada, de maneira padronizada, antes da cirurgia, no transoperatório, seis meses após a cirurgia e no último retorno do paciente ao ambulatório, para cada membro operado. Nas primeira e última ocasiões foi medida a amplitude de movimento do joelho com goniômetro e, em todas as avaliações, foram pesquisadas subjetivamente dor e queixas quanto à marcha.

A avaliação radiográfica pré-operatória constou de radiografia panorâmica ortostática nas projeções ântero-posterior e perfil, com filme de $130 \mathrm{~cm}$ e ampola de raios-X situada a $3 \mathrm{~m}$ do filme ${ }^{(12-14)}$. O paciente foi posicionado com as patelas direcionadas para frente, independentemente da posição dos pés. As medidas radiográficas seguiram o método de Paley et al ${ }^{(5,14)}$, com a seguinte sequiência: 1 . Traçado do eixo mecânico (reta definida entre o centro do quadril e o centro do tornozelo); 2. Medida da distância horizontal do eixo mecânico ao centro do joelho (centro das espinhas tibiais), em milímetros. Foram consideradas mau alinhamento distâncias maiores que $15 \mathrm{~mm}$ medialmente (varo) e $15 \mathrm{~mm}$ lateralmente (valgo), ao centro do joelho ${ }^{(10)}$. A amostra apresentou desvio médio nos pacientes com deformidade em valgo de 58,4mm (32mm-94mm) e, nas deformidades em varo, o desvio médio foi de $43,6 \mathrm{~mm}$ (23mm-65mm); 3. Determinação da presença de má orientação da articulação femoral distal. Isso é realizado pela medida do ângulo mecânico femoral distal lateral (mLDFA), definido pela intersecção do eixo mecânico do fêmur (linha entre o centro da cabeça femoral e o centro do joelho) com a linha da articulação distal do fêmur (linha tangencial aos côndilos lateral e medial). $\mathrm{O}$ valor normal desse ângulo situa-se entre $85^{\circ}$ e $90^{\circ}(5)$. Valores abaixo de $85^{\circ}$ indicam deformidade femoral em valgo e, maiores do que $90^{\circ}$, em varo. 
O valor médio pré-operatório do ângulo mecânico femoral distal lateral nesta série foi $74,7^{\circ}\left(70^{\circ}-80^{\circ}\right)$ para as deformidades em valgo e $108,3^{\circ}\left(120^{\circ}-100^{\circ}\right)$ para as deformidades em varo; 4 . Determinação da grandeza e do nível da deformidade femoral pela intersecção dos eixos mecânicos dos segmentos femorais proximal e distal. Esse ponto é definido como centro de rotação da angulação (CORA). No CORA, a deformidade apresenta apenas componente angular e a correção nesse nível restaura o eixo mecânico e a orientação articular normal. Se a correção for realizada em nível diferente ao CORA, a combinação de angulação e translação será necessária para a correção do eixo mecâni$\mathrm{co}^{(5,14)} ; 5$. Identificação das deformidades na região proximal da tíbia de modo semelhante ao utilizado para o fêmur, por meio da medida do ângulo medial proximal da tíbia, formado pela intersecção do eixo mecânico da tíbia com sua linha de orientação articular proximal (normal: $85^{\circ}-90^{\mathbf{o}(5,14)}$ ).

Foram realizadas radiografias na sala cirúrgica e, depois, a cada quatro semanas, sendo considerada consolidada a osteotomia que apresentasse continuidade óssea em pelo menos três córtices.

A avaliação radiográfica final foi realizada pelo menos seis meses após a retirada do fixador externo, seguindo os mesmos passos e técnica radiográfica da avaliação pré-operatória. Foram comparados, para cada paciente, os valores pré e pós-operatórios do desvio do eixo mecânico e do ângulo mecânico femoral distal lateral (mLDFA).

\section{Técnica cirúrgica}

Para a fixação definitiva da osteotomia foi utilizado um fixador externo híbrido com montagem modificada, construído com material de Ilizarov e fixador AO tubular ${ }^{(11,15)}$.

O procedimento era iniciado com a passagem de um fio de Kirschner de 1,8mm na metáfise distal do fêmur, paralelamente à linha articular do fêmur distal, com o auxílio do intensificador de imagens. Quando a cartilagem de crescimento estava presente, esse fio era colocado proximalmente a ela. A seguir, era instalado o anel do fixador externo de Ilizarov sobre esse fio de referência e realizada fixação adicional do anel com dois pinos de Schanz de 5,0mm, nas posições de 8 e 4 horas, observando alinhamento perpendicular do anel com o fêmur distal no plano sagital (figura 1a). Um pino de Schanz de 5,0mm era introduzido na região lateral do fêmur, em torno de $2,0 \mathrm{~cm}$ proximalmente ao local predeterminado para a osteotomia percutânea, por meio de acesso lateral no terço distal da coxa de $2 \mathrm{~cm}$ de comprimento, em média, com osteótomo, após múltiplas perfurações com broca de 2,5mm (figura 1b). Em seguida, era feita a translação do fragmento distal programada pelo estudo prévio associado à angulação (figura 1c), conectado o anel de Ilizarov ao pino de Schanz proximal com barra tubular modificada, e apertadas todas as conexões.

A correção do eixo mecânico era confirmada transoperatoriamente com intensificador de imagens, pelo posicionamento de um fio metálico flexível do centro da cabeça femoral ao centro do joelho. A confirmação da correção do eixo mecânico era realizada pela men-

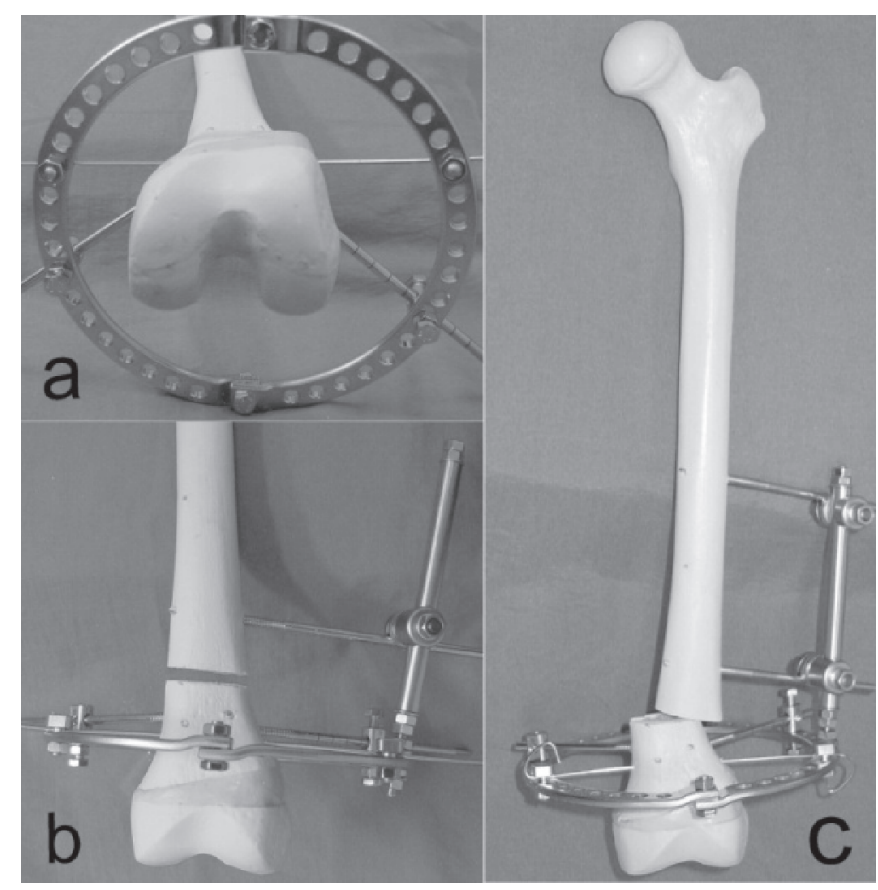

Figura 1 - Ilustração em modelo de fêmur dos detalhes técnicos de montagem do sistema de fixação. a) Fixação-padrão do anel metafisário, com o objetivo de evitar a transfixação do aparelho extensor; b) Osteotomia percutânea após a fixação inicial do fragmento proximal; c) Aspecto final da montagem, após correção da angulação e translação no foco da osteotomia. 
suração do mLDFA, em radiografia do fêmur em AP, ainda no período transoperatório.

Após obtenção do alinhamento satisfatório do eixo mecânico e mLDFA, eram acrescentados mais um ou dois pinos de Schanz de 5,0mm no fragmento proximal (figura 1c). Em casos selecionados (pacientes obesos ou esqueleticamente maduros) era acrescentada uma segunda barra tubular que conectou a barra lateral e a porção medial do anel, por meio de uma plataforma tubo-a-tubo, de modo a neutralizar forças de varização.

Não era utilizada imobilização no período pós-operatório e a reabilitação era supervisionada pelo setor de fisioterapia da instituição. Não houve apoio do membro operado nas primeiras quatro semanas e não era realizada dinamização do aparelho em caso nenhum. Após a retirada do fixador, os pacientes foram orientados a permanecer com carga parcial por mais quatro semanas, iniciando programa de reabilitação para ganho de movimento e fortalecimento muscular. Após esse período era permitida carga total progressiva.

\section{Análise estatística}

A distribuição normal das amostras foi verificada pelo teste de Kolmogorov-Smirnov e a comparação feita separadamente para as deformidades em varo, antes e depois da cirurgia e, para as deformidades em valgo, antes e depois da cirurgia, pelo teste " $t$ " de Student pareado.

O seguimento clínico médio foi de 17,7 meses (seis meses - 39 meses), com comparecimento de todos os pacientes às reavaliações programadas.

\section{RESULTADOS}

Os dados gerais da população estudada com os resultados das correções estão apresentados na tabela 1 .

Todas as osteotomias consolidaram-se, com tempo médio de três meses (variação de dois a cinco meses). Um paciente portador de displasia espondilometafisária apresentou fratura do fêmur após a retirada do fixador (caso no 4), que foi tratada com reinstalação do fixador híbrido. Neste caso, ocorreu a consolidação

TABELA 1

Dados gerais da população estudada e as correções obtidas

\begin{tabular}{|c|c|c|c|c|c|c|c|c|c|}
\hline \multirow[t]{2}{*}{ Indivíduos } & \multirow{2}{*}{$\begin{array}{c}\text { № } \\
\text { casos }\end{array}$} & \multirow[t]{2}{*}{ Gênero } & \multirow{2}{*}{$\begin{array}{l}\text { Idade } \\
\text { (anos) }\end{array}$} & \multirow{2}{*}{$\begin{array}{l}\text { Seguimento } \\
\text { (meses) }\end{array}$} & \multirow{2}{*}{$\begin{array}{l}\text { Consolidação } \\
\text { (meses) }\end{array}$} & \multicolumn{2}{|c|}{ Desvio eixo mecânico (mm) } & \multicolumn{2}{|c|}{ mLDFA (graus) } \\
\hline & & & & & & $\begin{array}{c}\text { Pré- } \\
\text { operatório }\end{array}$ & $\begin{array}{c}\text { Pós- } \\
\text { operatório }\end{array}$ & $\begin{array}{c}\text { Pré- } \\
\text { operatório }\end{array}$ & $\begin{array}{c}\text { Pós- } \\
\text { operatório }\end{array}$ \\
\hline 1 & 1 & $\mathrm{~F}$ & 20 & 38 & 3 & -44 & 0 & 80 & 93 \\
\hline 2 & 2 & $\mathrm{~F}$ & 10 & 37 & 2 & -94 & -24 & 72 & 87 \\
\hline 3 & 3 & $\mathrm{~F}$ & 7 & 6 & 3 & 23 & 0 & 100 & 94 \\
\hline 5 & 6 & M & 14 & 26 & 3 & 40 & 21 & 116 & 105 \\
\hline 6 & 7 & $\mathrm{~F}$ & 14 & 39 & 4 & -61 & -10 & 70 & 88 \\
\hline 7 & 8 & $\mathrm{~F}$ & 4 & 16 & 4 & -57 & -25 & 80 & 90 \\
\hline 8 & 9 & $M$ & 3 & 14 & 2 & -32 & -21 & 76 & 89 \\
\hline 10 & 14 & & 14 & 7 & 4 & 54 & 27 & 106 & 102 \\
\hline 11 & 15 & $\mathrm{~F}$ & 12 & 10 & 2 & -72 & -21 & 70 & 90 \\
\hline
\end{tabular}

Fonte: SAME HCFMRP-USP.

$\mathrm{F}=$ feminino; $\mathrm{M}$ = masculino; $\mathrm{D}$ = direito; $\mathrm{E}$ = esquerdo; $\mathrm{mm}$ = milímetro; $\mathrm{mLDFA}$ = ângulo lateral distal do fêmur (mecânico). 
após três meses, sem necessidade de utilização de enxerto ósseo ou outro tipo de intervenção.

$\mathrm{Na}$ avaliação clínica final, nenhum paciente necessitou apoio para a deambulação e não apresentava queixas de dor. A mobilidade articular do joelho apresentou-se normal e restaurada aos valores pré-operatórios na avaliação após seis meses, em todos os casos. Não houve caso de infecção profunda durante o tratamento, sendo que em um paciente foi usado antibiótico profilático por quatro semanas, por ser portador de neutropenia cíclica. Não houve caso de perda de fixação ou falha mecânica do fixador e nenhum paciente desenvolveu qualquer tipo de lesão neurológica periférica imediata ou tardia.

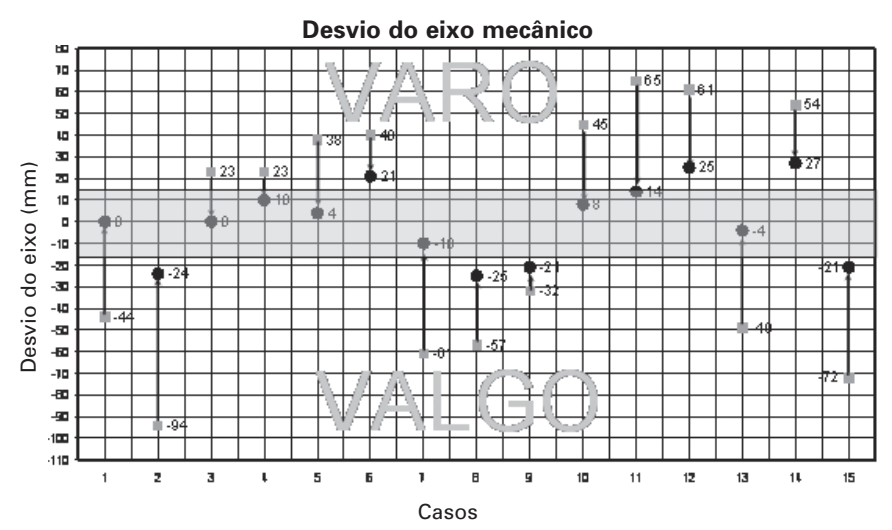

Figura 2 - Desvio do eixo mecânico, em milímetros, do centro do joelho. Os valores pré-operatórios estão representados por quadrados e os pós-operatórios por círculos, para cada membro tratado. A faixa em cinza representa o intervalo considerado normal.
$\mathrm{Na}$ avaliação após seis meses, o eixo mecânico foi completamente restaurado em oito das 15 extremidades, com valor médio nos pacientes com fêmur valgo de $15 \mathrm{~mm}(0 \mathrm{~mm}-25 \mathrm{~mm}$; $\mathrm{p}<0,001)$ e de $13,6 \mathrm{~mm}$ ( $0 \mathrm{~mm}$ $27 \mathrm{~mm} ; \mathrm{p}<0,001)$ nas deformidades em varo. O ângulo mecânico femoral distal lateral médio final foi $89,6^{\circ}$ $\left(88^{\circ}-93^{\circ} ; \mathrm{p}<0,001\right)$ nas deformidades em valgo do fêmur e $94,8^{\circ}\left(87^{\circ}-105^{\circ} ; \mathrm{p}=0,004\right)$ nas deformidades em varo. Os valores individuais do eixo mecânico e ângulo mecânico femoral distal lateral pré e pós-operatório para cada membro estão ilustrados nas figuras 2 e 3 . A figura 4 ilustra um caso tratado pelo método.

Não houve caso de alteração na consolidação da osteotomia tibial quando realizada em associação com a

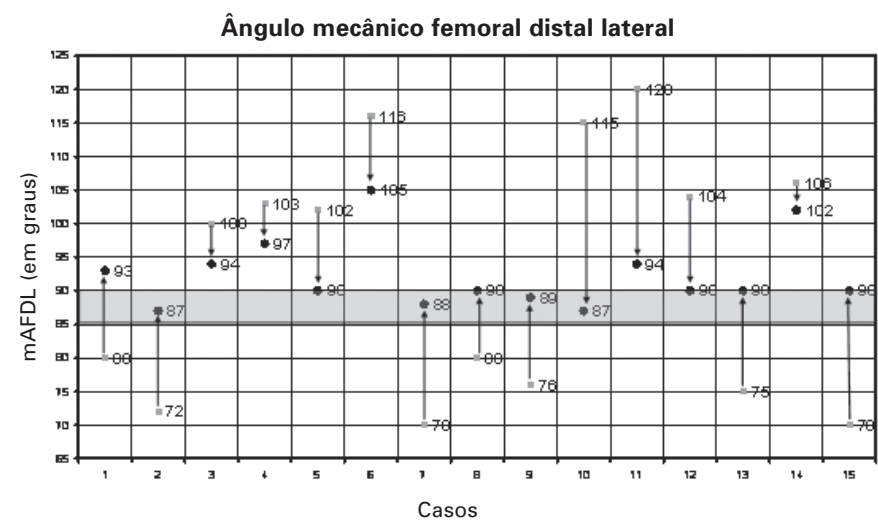

Figura 3 - Ângulo mecânico femoral distal lateral, medido em graus. Os valores pré-operatórios estão representados por quadrados e os pós-operatórios por círculos, para membro tratado. A faixa em cinza representa o intervalo considerado normal.
Figura 4 - Exemplo de correção de deformidade complexa decorrente de raquitismo hipofosfatêmico, abrangendo a região distal do fêmur, tíbia e pé esquerdo: a) pré-tratamento; b) aspecto clínico após correção da deformidade femoral com o fixador externo híbrido e da tíbia com placa; c) alinhamento clínico final.
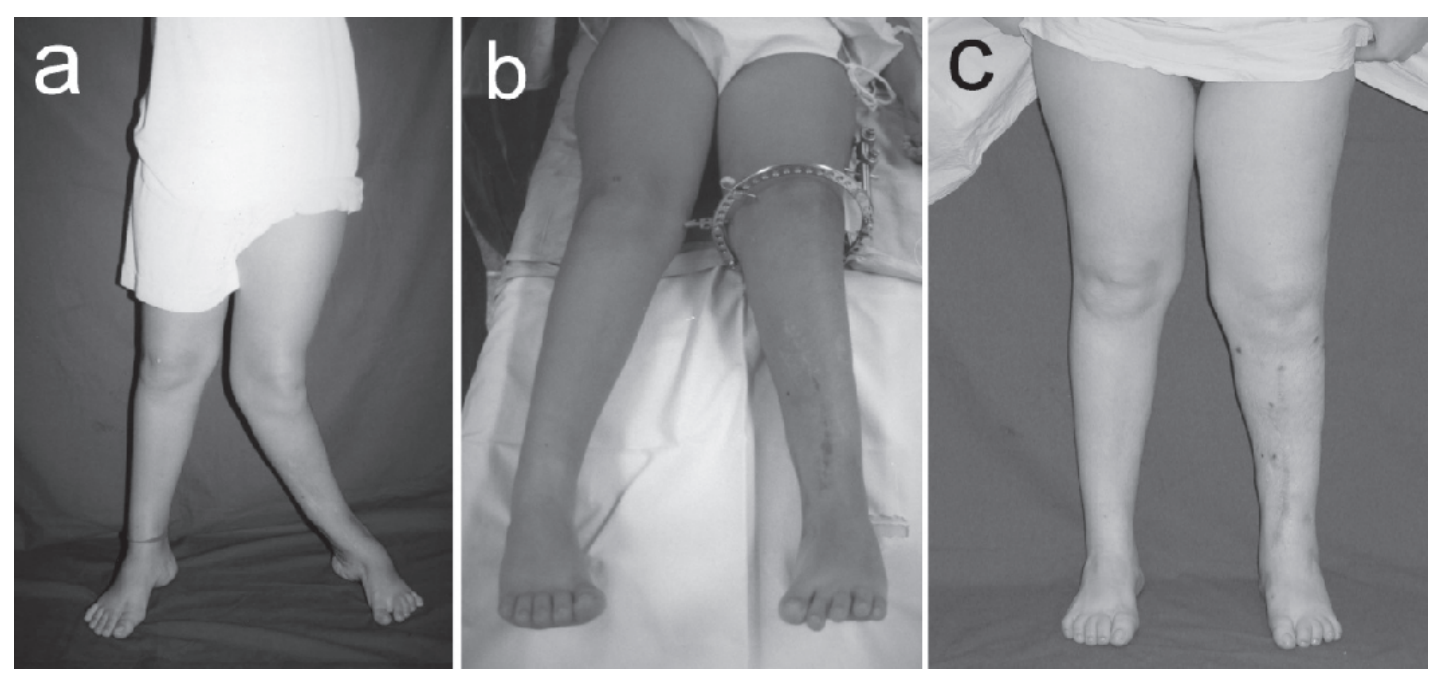
fixação externa para o fêmur e o regime pós-operatório desses pacientes também não foi diferente dos demais.

\section{DISCUSSÃO}

As principais vantagens teóricas do uso do fixador externo com montagem híbrida é não provocar encurtamento ósseo indesejado, permitir correção das deformidades em varo e valgo com a mesma técnica cirúrgica, envolver dissecação limitada das partes moles e apresentar aspecto estético bom, com cicatrizes pequenas e alinhamento adequado do membro.

Entretanto, a maior característica do método é fornecer estabilidade adequada à osteotomia, diferentemente dos fixadores unilaterais utilizados em condições semelhantes ${ }^{(5,9)}$. Esse aspecto pôde ser apreciado com a análise dos 15 fêmures tratados com este método, pois não houve perda da fixação com desvios secundários em ante ou recurvato. Outro indicador da estabilidade foi a consolidação, que ocorreu em todos os casos. Além disso, a técnica permite inclusive correção simultânea de pequenos desvios do plano sagital do fêmur distal, com o cuidado de fixar o anel de Ilizarov paralelamente à orientação articular do fêmur distal no plano sagital.

Nossa casuística é composta por pacientes com grande variação da faixa etária, grande variação do grau de deformidade e de etiologias. Essas características, se por um lado contribuem para heterogeneidade do grupo, por outro refletem exatamente a população que necessita este tipo de correção. Exceto por um paciente de 17 anos em que ocorreu fratura após a retirada do fixador, não houve complicação importante. Mesmo nesse caso a recolocação do fixador permitiu a consolidação, o que reflete estabilidade suficiente e boa superfície de contato ósseo, apesar de não ter sido feita a osteotomia em cúpula, como sugerem alguns autores $^{(5,9)}$.

O procedimento, tecnicamente simples, envolve, no entanto, planejamento prévio minucioso, com estudo do eixo mecânico e localização do(s) centro(s) de rotação da(s) deformidade(s). Nossa impressão é semelhante à dos relatos de outros autores quer usaram o fixador híbrido $\mathrm{AO}^{(11,15)}$. Apesar de não se dispor de facilidades que o aparelho AO apresenta (construção em fibra de carbono, junta universal entre a barra e o anel, possibilidade de colocação dos fios e pinos em qualquer posição e não apenas onde existem orifícios, como no material de Ilizarov), não houve complicações com a utilização do fixador modificado.

Outro fator relevante foi a boa tolerância ao fixador apresentada pelos pacientes. Embora nossa metodologia não tenha feito comparação dessa técnica com outra, a deambulação precoce e carga parcial a partir da quarta semana pós-operatória demonstraram a boa tolerância dos pacientes ao método.

$\mathrm{Na}$ avaliação dos resultados obtidos, sete extremidades em sete indivíduos apresentaram eixo mecânico subcorrigido na avaliação final, apesar da melhora substancial, quando comparados com os valores pré-operatórios. Em quatro casos, a causa do mau alinhamento residual foi a hipocorreção de deformidade em valgo da tíbia, visto que o ângulo mecânico femoral distal lateral estava dentro da faixa normal; em dois casos, ocorreu hipocorreção da deformidade femoral, com o ângulo mecânico femoral distal lateral fora da faixa normal; e, em um caso, a deformidade em varo residual ocorreu devido à coxa vara não corrigida. $\mathrm{O}$ ângulo mecânico femoral distal lateral retornou para a faixa normal em nove dos 15 fêmures. Dos seis casos com mAFDL anormal, quatro estavam com o eixo mecânico nos limites normais, onde foi propositalmente causada pequena hipocorreção ou hipercorreção para compensar deformidades pequenas na tíbia. Houve dois casos de hipocorreções da deformidade femoral com eixo mecânico anormal, que ocorreram no início de nossa experiência com a técnica.

Autores recomendam a correção de todas as deformidades para restaurar completamente o eixo mecânico e a orientação das articulações dos membros inferiores $^{(1,4-5,10)}$. Aceitamos nesta série pequenas compensações, por dois motivos: na faixa etária pediátrica, o alinhamento dos membros inferiores está em constante mudança, principalmente em doenças metabólicas e displásicas, que tendem a estabilizar-se apenas após a maturação esquelética; o segundo motivo é que alguns desses pacientes já tinham sido submetidos a vários procedimentos cirúrgicos e recusaram tra- 
tamentos mais extensos ou demorados. É nossa impressão, após a análise dos resultados, que é muito importante a medida do mAFDL transoperatoriamente, e não somente a avaliação do eixo mecânico, principalmente quando se corrigem simultaneamente deformidades em outros níveis.

\section{CONCLUSÃO}

A técnica de correção do desvio da região distal do fêmur, com osteotomia distal compensada pela translação do fragmento, foi adequada para restaurar o eixo mecânico do membro inferior. O fixador externo híbrido com a montagem modificada foi eficiente para manter as correções obtidas no momento da cirurgia e propiciar a consolidação óssea.

\section{REFERÊNCIAS}

1. Tetsworth K, Paley D. Malalignment and degenerative arthropathy. Orthop Clin North Am. 1994;25(3):367-77.

2. Johnson F, Leitl S, Waugh W. The distribution of load across the knee. A comparison of static and dynamic measurements. J Bone Joint Surg Br. 1980;62(3):346-9.

3. McKellop HA, Sigholm G, Redfern FC, Doyle B, Sarmiento A, Luck JV Sr. The effect of simulated fracture-angulations of the tibia on cartilage pressures in the knee joint. J Bone Joint Surg Am. 1991;73(9):1382-91.

4. Tetsworth KD, Paley D. Accuracy of correction of complex lower-extremity deformities by the Ilizarov method. Clin Orthop Relat Res. 1994;(301):102-10.
5. Paley D. Principles of deformity correction. New York: Springer; 2002.

6. Healy WL, Anglen JO, Wasilewski SA, Krackow KA. Distal femoral varus osteotomy. J Bone Joint Surg Am. 1988;70(1): 102-9.

7. Terry GC, Cimino PM. Distal femoral osteotomy for valgus deformity of the knee. Orthopedics. 1992;15(11):1283-9.

8. Mathews J, Cobb AG, Richardson S, Bentley G. Distal femoral osteotomy for lateral compartment osteoarthritis of the knee. Orthopedics. 1998;21(4):437-40.

9. Paley D. Herzenberg JE, Bor N. Fixator-assisted nailing of femoral and tibial deformities. Tech Orthop. 1997;12:26-75.

10. Gugenheim JJ Jr, Brinker MR. Bone realignment with use of temporary external fixation for distal femoral valgus and varus deformities. J Bone Joint Surg Am. 2003;85-A(7):1229-37.

11. Faria LOM, Motta Filho GR, Storino HP, Areas RV. Um método simples para montagem de um fixador externo híbrido. Rev Bras Ortop. 1999;34(7):435-8.

12. Moreland JR, Bassett LW, Hanker GJ. Radiographic analysis of the axial alignment of the lower extremity. J Bone Joint Surg Am. 1987;69(5):745-9.

13. Wright JG, Treble N, Feinstein AR. Measurement of lower limb alignment using long radiographs. J Bone Joint Surg Br. 1991; 73(5):721-3.

14. Paley D, Herzenberg JE, Tetsworth K, McKie J, Bhave A. Deformity planning for frontal and sagittal plane corrective osteotomies. Orthop Clin North Am. 1994;25(3):425-65.

15. Remiger AR, Miclau T, Neuer W. A simple technique for creating hybrid fixators using a modified AO single adjustable clamp. J Orthop Trauma. 1997;11(1):54-6.

\section{Declaração de inexistência de conflitos de interes-} se: Não houve auxílio à esta pesquisa e não há conflitos de interesse conforme estabelecido nas normas da $R B O$. 